\title{
Transformerless SoC-based current control switching battery charger for e-vehicle: design and analysis
}

\author{
Purnomo Sidi Priambodo, ${ }^{1, *}$, Yosua Adriadi ${ }^{1}$ and Taufiq Alif Kurniawan ${ }^{1}$ \\ ${ }^{1}$ Department of Electrical Engineering, Universitas Indonesia, Depok 16424, Indonesia
}

\begin{abstract}
Electric-based vehicles become a necessity in the future to dramatically reduce the effects of pollution. There are various devices involve in the operation of electric vehicles, one of which is a battery charger. This paper discusses the design steps, circuit and ripple performance analysis associated with building a battery charger system. The analysis shows the differences between RL and RC circuits in controlling the output voltage average and the ripples. It shows how RLC mixed circuit improve performance in both controlling the output voltage average and the ripple suppression.
\end{abstract}

\section{Introduction}

Global warming effects have driven nations around the world become more aware that the reduction of pollution must be resolved as soon as possible and suppress it to the lowest level. Supported by the mature of electric and electronics technology, it is predicted that in the next 50 years the combustion-based vehicles will disappear or be transformed into a much more efficient and non-pollutive electric-based vehicles [1]. It is not simple to replace $100 \%$ of fossil-fuel based vehicles to the $100 \%$ of electric based vehicles. It requires preparation of various infrastructures to support this replacement. In between, the hybrid vehicle technology has emerged to bridge the implementation of $100 \%$ electric based vehicles. There is a report that the efficiency of hybrid vehicle is almost 2-3 times compared to the full-combustion vehicles [2]. Various automotive industry has come to enliven the market with a variety of electric vehicle products, from small size of motorcycles, cars and even buses.

Based on technology trends, electric vehicles can be grouped based on (1) battery technology, (2) fuel cell technology and (3) mini nuclear reactor technology [3]. Of the three technologies, only the two early technologies are predicted will compete as the main technology supporting electric vehicles in the future, because nuclear technology is considered not environmental clean. Of the first two technologies, namely battery-based and fuel-cell, both have advantages and disadvantages. One disadvantage of battery-based electric vehicles compared to fossil-fueled vehicles and fuel-cell-based electric vehicles is the time needed to charge the battery for longer vehicle mileage. In addition, the weight of the battery itself is much heavier when compared to other fuel-based vehicles. However, due to the longer maturity of battery technology and getting lower price, the battery-based vehicles become more favorable in the last decade compared to fuel-cell based electric vehicles. Hence, most of the existing electric vehicles in the market nowadays are based on battery technology. So, it became an interest to develop some components of battery-based electric vehicles, one of which is a battery charger.

Battery charger becomes an important instrument component in battery-based electric vehicles. There are at least three main parameters in battery charging process will be discussed here, i.e. battery capacity, battery charging speed and filling method. The larger the battery capacity, the more energy backup of the vehicle and the more distance it can reach. However, the consequence, for charging electricity to large battery size will require more time than the small capacity one. Battery charging speed is one of the disadvantages of the first generation batterybased electric vehicles. Battery charging speed limits are more determined by the battery character limitations than the charger current delivery capability. Charger can be made for any current capacity, however, battery speed is generally determined by Charge Rate (CR) battery in Amperes. For instant, battery capacity of $12 \mathrm{~V} 65 \mathrm{Ah}$ battery, if it has CR 13A, it will take about 5 hours to fully charge the battery. For CR 65 A, it will take 1 hour [4]. In general, $\mathrm{CR}$ is limited by the internal resistance of the battery at charging state, which is a function of state of charge (SoC). Hence, the ability of CR is a function of the SoC. Generally, the maximum $\mathrm{CR}$ is specified by the battery manufacturer. Obviously, SoC is divided into 3 regions. SoC from 0 to $70 \%$ is called as empty, $70 \%-90 \%$ is called half full and $90 \%$ to full is called full condition. In an empty $\mathrm{SoC}$ condition, the internal charging resistance of the batteries is relatively low compared to the full SoC condition. So, in the condition of the empty SoC is possible to do large CR (charging current) with relatively small heat emissions, compared to the same CR, but at full SoC condition. In order to avoid excessive heat emission, it must be arranged $\mathrm{CR}$ in accordance with the internal charging resistance battery as a function of SoC.

*Corresponding author: p.s.priambodo@ieee.org 
This paper discusses the design steps, circuit and ripple performance analysis in order to build a transformerless SoC-based current control switching battery charger system for 100 VDC battery system. The analysis shows the differences between RL and RC circuit in controlling the output voltage average and the ripples. It shows how RLC mixed circuit improves the charging performance in both controlling the output voltage average and ripple suppression.

\section{Theoretical, design and analysis}

We designed this battery charger with several features, such as: transformerless, soft-switch and electronic switching [5-8]. As is common in modern battery chargers, this system does not use transformers for stepup or step down to the desired DC charging voltage for compact and low cost reason. This system directly performs the rectifying process of $\mathrm{AC}$ source voltage i.e. electric utility $220 \mathrm{VAC} 50 \mathrm{~Hz}$. The desired DC charging voltage and current are adjusted by the electronic switching system with the duty-cycle arrangement. In our developed battery charger system, the duty cycle switching control is conducted by the PWM generator circuit, which gets input control from the value of the SoC battery being charged.

\subsection{Battery charger circuit}

The following Fig-1 shows the overall schematic block of the battery charger system.

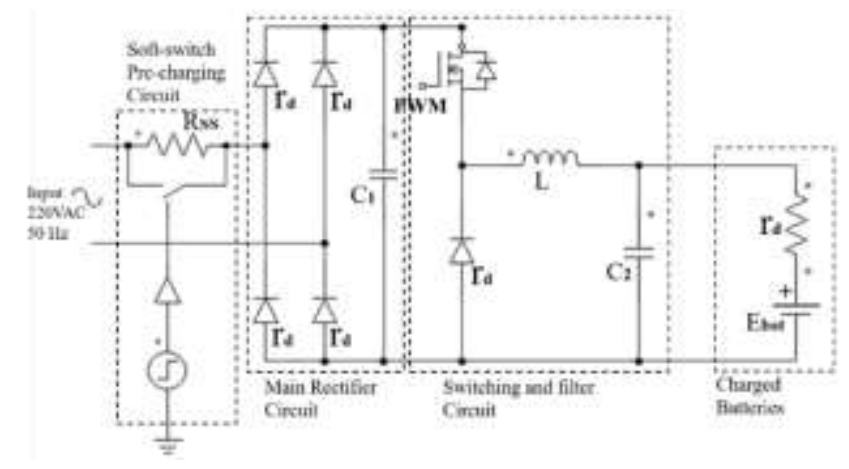

Fig. 1. Battery charger circuit.

\subsection{Soft-switch circuit:}

Soft-switch pre-charging circuit is used to avoid rush current at turn-on time, the beginning of charging process and capacitor $C_{l}$ at main rectifier still empty. At the turnon time, the $C_{I}$ capacitor is charged through the $R_{S S}$ (softswitch resistance), such that the charging current can be limited. After some time (2-3 seconds), $C_{l}$ is filled up at certain sufficient safety level, the $R_{S S}$ is then by-passed by the controlled relay Soft-switch pre-charging circuit. The Soft-switch circuit is shown on Fig-2 below.

Delay switching time to bypass pre-charging resistor is determined by time constant $\tau=R_{l} C_{l}$ of Fig- 2 . The delay is about 2 to 3 seconds which allows the rectifier main capacitor $C_{l}$ on Fig-1 to be partially filled. To meet the $\tau$ (2 to 3 ) second delay the value of $R_{l}$ and $C_{l}$ on Fig2 must meet the requirement.

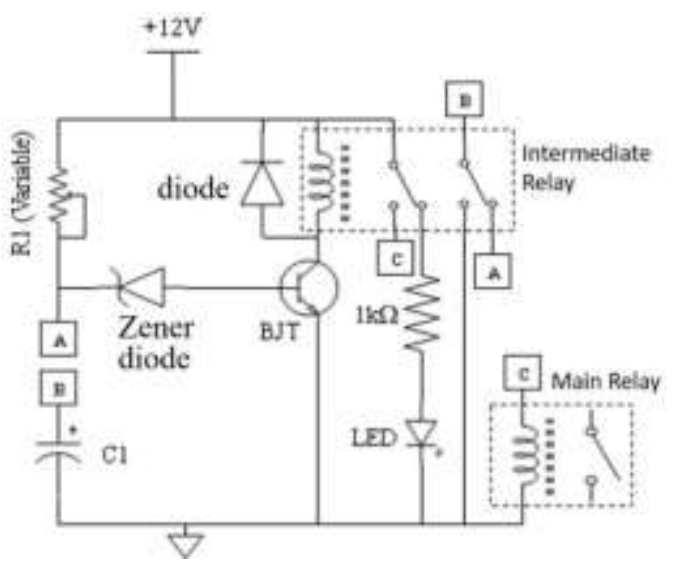

Fig. 2. Soft-switch circuit.

\subsection{Main rectifier circuit}

The output voltage of the main rectifier circuit is the input for the switching circuit. It should be designed in such a way that the output voltage ripple as low as possible. Therefore, the design of the filter capacitor on the main rectifier circuit should be based on its maximum load. The electric circuit model of the main rectifier circuit is in the following Fig-3, where the switching circuit is represented as $R_{L o}$ :

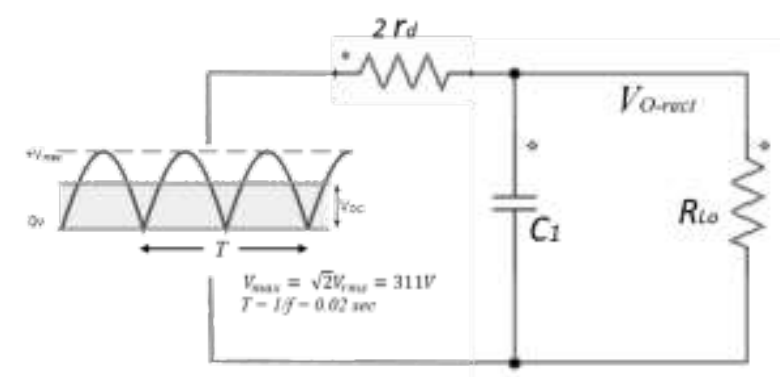

Fig. 3. Electric circuit model for the main rectifier circuit.

Output $V_{O-r e c t}(\mathrm{t})$ or $V_{c}(t)$ as a with and without capacitor $C_{1}$ is illustrated in the following Fig-4.

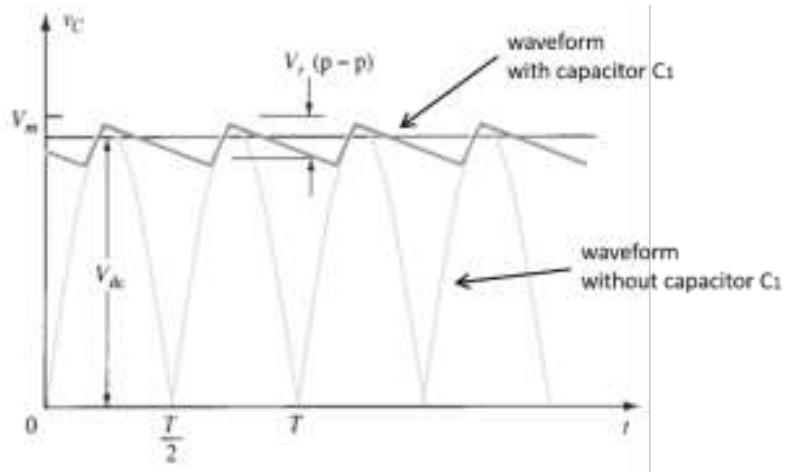

Fig. 4. Full-wave rectifier output ripple noise illustration.

The Output of bridge rectifier diode is a full-wave rectifier, if not filtered by capacitor, then: 
$V_{D C}=\frac{R_{L o}}{2 r_{d}+R_{L o}} \frac{2}{\pi}\left(V_{\max }-0.7\right)$, where $r_{d}$ and $R_{L o}$ are static diode resistance and load resistance, respectively. 2 $r_{d}$ means 2 series diode resistances in a charging loop. If it is filtered by capacitor $C_{1}$ then:

$V_{D C}=\frac{R_{L o}}{2 r_{d}+R_{L o}}\left(V_{\max }-0.7\right)$. Where the value of $V_{\max }$ is $\sqrt{2} V_{r m s_{-}}$rms or $311-\mathrm{V}$. In order to suppress the output ripple, it requires that time constant $R_{L o} C_{1} \gg 0.5 \mathrm{~T}$, where $\mathrm{T}$ is the time period of the electric source $(0.02 \mathrm{sec})$. The peak to peak ripple magnitude to the maximum voltage output of full-wave rectifier filtered by $C_{1}$ is written in the following formula:

$$
\text { Ripple } \%=\frac{1}{2 f R_{L o} C_{1}} \times 100 \%
$$

For a half-wave rectifier filtered by $C_{1}$ is written as:

$$
\text { Ripple } \%=\frac{1}{f R_{L_{0} C_{1}}} \times 100 \%
$$

where $f$ is $50 \mathrm{~Hz}$ and $R_{L o}$ is the equal load resistance.

\subsection{Switching circuit}

The switching circuit is shown in detail in Fig-5 below:

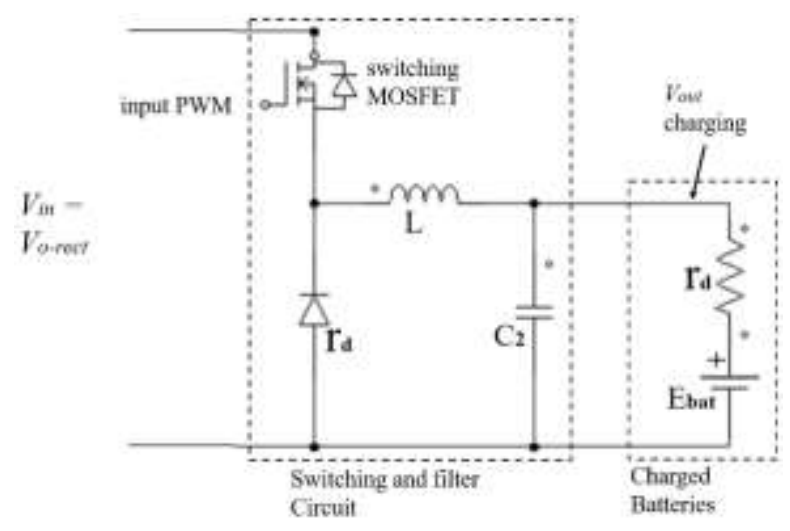

Fig. 5. Single MOSFET switching circuit for DC to DC converter.

Furthermore, the $V_{D C}$ voltage generated by the main rectifier circuit will be set in such a way as to be able to charge the battery with a controlled current. To control charging voltage and current, the switching method is applied using a single MOSFET, by adjusting the switching frequency and duty cycle. The switched current then filtered by using $R L C$ circuit or called Snubber circuit. The switching driver circuit is not discussed here. In later derivation, we show how using higher frequency switching will help to reduce the size of $L$ and $C_{2}$ component values compared to the one using a lower frequency for the same level of reduction noise ripple. In other words, the use of higher frequency is effective for suppressing ripple noise. The duty cycle setting is to control the output $V_{D C}$, such that the output current can be controlled.

In this design, we use $10-\mathrm{kHz}$ switching frequency that is considered to be optimal. How the Switching and filter circuit (often referred to as Snubber circuit) works and how the system performs, all is analyzed in the following description.

The battery load can be illustrated as $R_{\text {Load }}$, then the $R_{\text {Load }} L C$ filter circuit can be described as two synergistic filters, $R_{\text {Load }} L$ and $R_{\text {Load }} C$. Both filters have similarity properties in controlling the amount of ripple noise, but different in controlling the output voltage of Vo. Briefly we describe the similarities and differences between those two filter types. First, we analyze the filter that contains only the $L$ and $R_{\text {Load }}$ components ( $R_{\text {Load }}$ represents the battery load) like the following Fig-6. It is given the input parameters of switching voltage of $V$, frequency $f$ (period $T)$ and duty cycle of $d$, then $V o(t)$ is illustrated in Fig-7.

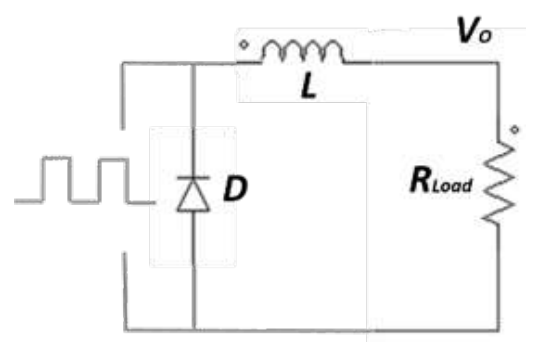

Fig. 6. $R L$ Filter.

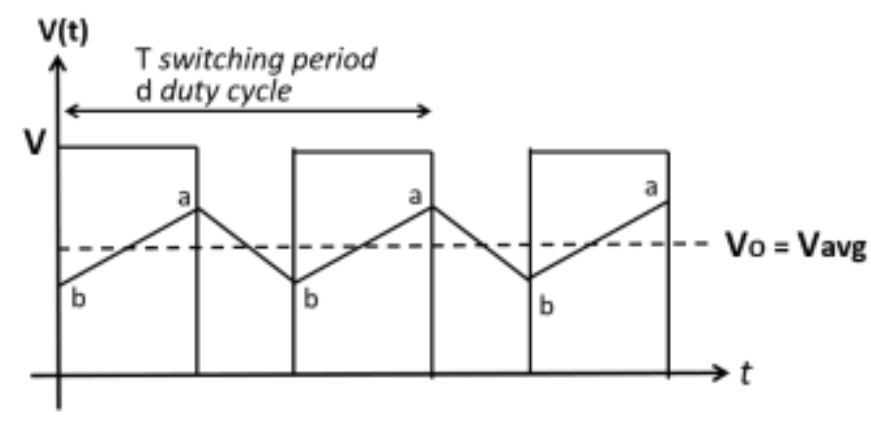

Fig. 7. $V(t)$ of RL Filter.

Moreover, $R_{\text {Load }}$ is named as $R$. Note that for Fig-7, line $b$ to $a$ is governed by $\left(1-e^{-\frac{R}{L} d t}\right)$ term, while line $a$ to $b$ is governed by $\left(e^{-\frac{R}{L}(1-d) t}\right)$ term. The maximum ripple value $a$ and the lowest $b$ of $R L$ filter circuit of Fig-6 and -7 are:

$$
\begin{aligned}
& a=V \frac{1-e^{-\frac{R}{L} d T}}{1-e^{-\frac{R}{L} T}} \\
& b=V \frac{e^{-\frac{R}{L}(1-d) T}\left(1-e^{-\frac{R}{L} d T}\right)}{1-e^{-\frac{R}{L} T}}
\end{aligned}
$$

For $\quad \frac{R}{L} d T \ll 1$, then $e^{-\frac{R}{L} d T} \cong 1-\frac{R}{L} d T+\left(\frac{R}{L} d T\right)^{2}$, moreover, we can rewrite:

$$
a=V \frac{d-\frac{\frac{R}{L} T D d^{2}}{2}}{1-\frac{R}{\frac{R}{2}}}
$$

and

$$
b=V \frac{d-\frac{\frac{R}{L} T(2-d) d}{2}}{1-\frac{\frac{R}{L} T}{2}}
$$

Output voltage is the average of $a$ and $b$ :

$$
V_{O}=V_{\text {avg }}=\frac{a+b}{2}=V \cdot d
$$


The ripple of $R L$ filter is:

$$
V_{\text {rip }}=\frac{(a-b)}{V_{\text {avg }}} \times 100 \%=\frac{2 \frac{R}{L} T}{2-\frac{R}{L} T} \times 100 \%
$$

Eq-7 shows that $R L$ filter control the average output voltage is linear to the duty cycle d, while Eq- 8 shows that the ripple is almost linear to $\frac{R}{L} T$ value, for $\left(\frac{R}{L} d T<1\right)$. Smaller $\frac{R}{L}$ ratio and $T$ will reduce the ripple.

If the filter consists only $C$ and $R_{\text {Load }}(R)$ (where $R_{\text {Load }}$ or $R$ represents battery load) as shown in the following Fig-8, where switching with voltage $V$, frequency $f$ (period $T$ ), duty cycle $d$ and $V o(t)$ are illustrated on Fig-9.

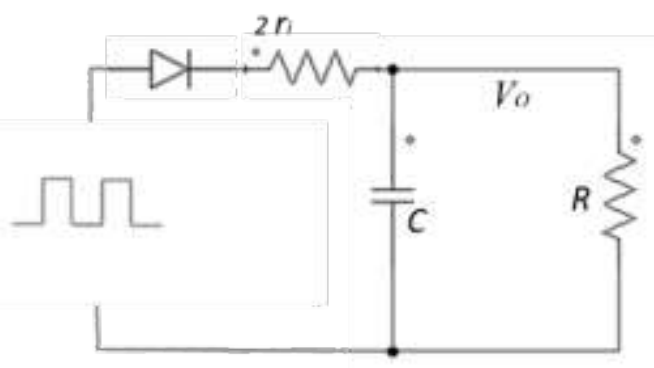

Fig. 8. RC Filter.

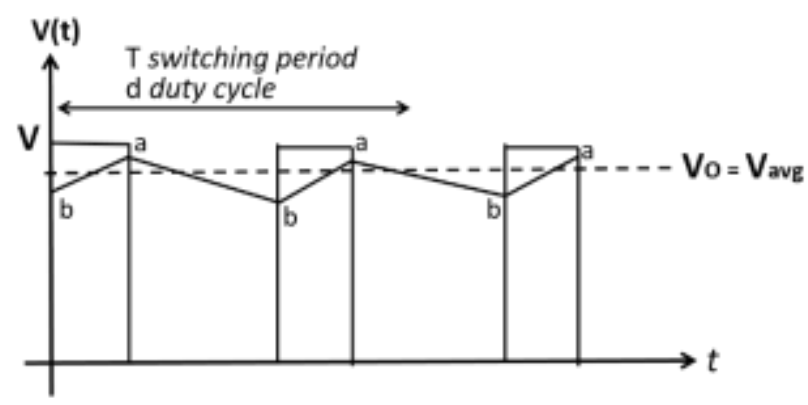

Fig. 9. $V(t)$ of $R C$ Filter.

On Fig-9, note that line $b$ to $a$ is governed by $\left(1-e^{-\frac{d t}{r_{d} C}}\right)$ term, while line $a$ to $b$ is governed by $\left(e^{-\frac{(1-d)}{R C} t}\right)$ term. The maximum ripple value $a$ and the lowest $b$ of $R C$ filter circuit of Fig-8 and -9 are:

$$
\begin{aligned}
& a=V \frac{1-e^{-\frac{1}{r_{i} C} d T}}{1-e^{-\left(\frac{1}{R C}(1-d)+\frac{1}{r_{i} C} d\right) T}} \\
& b=V \frac{e^{-\frac{1}{R C}(1-d) T}\left(1-e^{-\frac{1}{r_{i} C} d T}\right)}{1-e^{-\left(\frac{1}{R C}(1-d)+\frac{1}{r_{i} C} d\right) T}}
\end{aligned}
$$

For $\frac{1}{R C} d T \ll 1$, then $e^{-\frac{1}{R C} d T} \cong 1-\frac{1}{R C} d T+\left(\frac{1}{R C} d T\right)^{2}$, and we can rewrite:

$$
\begin{aligned}
& a=V \frac{1}{1+\frac{r_{i}}{R_{1}-\frac{d T}{r_{i} C}}} \\
& b=a\left\{1-\frac{1}{R C}(1-d) T\right\}
\end{aligned}
$$

Output voltage is the average of $a$ and $b$ :

$$
V_{O}=V_{a v g}=\frac{a+b}{2}=a\left\{1-\frac{1}{2} \frac{(1-d) T}{R C}\right\}=a\left\{1-\frac{1}{2} \frac{(1-d)}{f R C}\right\}
$$

For $\frac{(1-d)}{R C} T \rightarrow 0$ and $r_{i} \ll R, V_{O}=V_{a v g}=V$

The ripple of $\mathrm{RC}$ filter is:

$$
V_{\text {rip }}=\frac{(a-b)}{V_{\text {avg }}} \times 100 \%=\frac{\frac{(1-d)}{R C} T}{1-\frac{1(1-d) T}{2 R C}} \times 100 \%
$$

By assuming $\frac{(1-d)}{R C} T \rightarrow 0$ and $r_{i} \ll R$; then the ripple is

$$
V_{\text {rip }} \cong \frac{(1-d)}{R C} T \times 100 \%=\frac{(1-d)}{f R C} \times 100 \%
$$

Where $f=1 / T$ is the switching frequency.

Eq-14 shows that RC filter cannot be able to control the average output voltage linear to the duty cycle. While Eq16 shows that the ripple is close to $\frac{(1-d)}{R C} T$ value, for $\frac{(1-d)}{R C} T \ll 1$. Smaller $\frac{(1-d)}{R C}$ ratio and $T$ will reduce the ripple.

Hence, we can conclude that to control the output voltage and to minimize the ripple effectively, we should combine $R L$ and $R C$ in a $R L C$ circuit called snubber filter to suppress switching transient. $R L$ circuit function is both, to filter ripple as well as averaging the output voltage based on duty cycle, while $R C$ circuit function to filter the ripple only. If both are combined as in Fig-5 above, then we will get the output filter that is $V_{o}$ as written in Eq-7:

$$
V_{o}=V_{a v g}=V \cdot d
$$

It shows that $R L$ filter can control the output linear to the duty cycle $d$.

While the output ripple performance is cascading results of both filter types, i.e. $R L$ and $R C$.

By assuming $\frac{R}{L} T \& \frac{(1-d)}{R C} T \rightarrow 0$ and $r_{i} \ll R$; then the ripple is:

$$
V_{\text {rip }} \cong\left\{\frac{2 \frac{R}{L} T}{2-\frac{R}{L} T} \times \frac{(1-d)}{R C} T\right\} \times 100 \%
$$

\section{Conclusions}

We have designed and theoretically analyzed a battery switching charger system. As discussed above, the output voltage setting and subsequent current charging are controlled by the PWM switching generator, which receives the input from the $\mathrm{SoC}$ of the charged battery. From the analysis of the circuit above, we conclude that $R L$ serves to output averaging and simultaneously filtering the ripple, while $R C$ only works for filtering the ripple only. If both $R L$ and $R C$ are used in a single circuit $R L C$ Snubber filter, we will get overall high performance in controlling voltage and current output, while simultaneously suppressing ripple noise due to switching effectively. 
This research is funded by Hibah Publikasi Internasional Terindeks Untuk Tugas Akhir Mahasiswa (Hibah PITTA) TA 2018, 2507/UN2.R3.1/HKP.05.00/2018, Universitas Indonesia.

\section{References}

1. M.G. Neacsu, V.S. Stanciu, G.V. Risnoveanu, S.S. Matei and C. Nicolescu, IEEE Xplore, DOI: 10.1109/EV.2017.8242098, Bucharest, Romania 5-6 Oct. 2017

2. Deepak Somayajula, Andrew Meintz and Mehdi Ferdowsi, IEEE Vehicular Technology Magazine, Vol. 4 Issue 2 2009, pp. 65 - 72, 26 May 2009

3. M.V. Ramana, IEEE Spectrum Apr 27, 2015

4. Purnomo Sidi Priambodo, Wahyudi Purnomo, Aries Subiantoro, Abdul Muis and Feri Yusivar IEEE Xplore, DOI: 10.1109/rICT-ICeVT.2013.6741492

5. Liang-Rui Chen; Hsu, R.C. and Chuan-Sheng Liu, Industrial Electronics, IEEE Transactions on, Vol. 55 Issue. 10, pp. 3692-3701, 2008.

6. Liang-Rui Chen, IEEE Transactions on Industrial Electronics, Vol.54 Issue 1, pp. 398-405, 2007

7. Purnomo Sidi Priambodo, Didik Sukoco, Wahyudi Purnomo, Harry Sudibyo and Djoko Hartanto, INTECH open publication, pp. 327-351, March 2013, ISBN 978-953-51-1003-3, www.intechopen.com

8. Muhammad H. Rashid, Pearson India; 4th edition (2017), ISBN-13: 978-9332584587 